\title{
La synthèse protéique commence dans le noyau
}

Olivier Bensaude

$>$ Chez les bactéries telles qu'Escherichia coli, les ribosomes s'associent aux ARNm naissants et commencent à les traduire en polypeptide avant que la transcription ne soit achevée. II en résulte un couplage entre ces deux opérations. Ainsi, la manière dont s'effectue la transcription détermine la stabilité et la traductibilité des ARNm. Inversement, la traductibilité des ARNm détermine l'efficacité de leur transcription et leur stabilité. Jusqu'en 2001 , l'existence d'un tel couplage chez les eucaryotes semblait n'être qu'une hypothèse d'école. En effet, les cellules eucaryotes se distinguent des procaryotes par une compartimentation de l'espace intracellulaire en cytoplasme et noyau. Or, tous les manuels de biologie nous enseignent que le noyau est le siège de la transcription des ARN pré-messagers, puis de leur maturation en ARN messagers, alors que la synthèse protéique s'effectue dans le cytoplasme après exportation des ARNm hors du noyau [1]. Pourtant plusieurs études publiées dans les années 1970 avaient suggéré, sans convaincre, l'existence d'une synthèse protéique nucléaire. La question s'est reposée dans les années 1990, pour rendre compte d'un «contrôle de qualité» des ARNm avant leur exportation hors du noyau. Des mutations, à l'origine des codons «non-sens» ou stop, affectent l'épissage, et la dégradation des ARN pré-messagers. Or comment détecter la présence d'un codon non-sens dans un ARN nucléaire si celuici n'est pas traduit en polypeptide $(\rightarrow)$ ? On doit à F. Iborra et à P. Cook d'avoir réactivé l'hypothèse d'une synthèse protéique nucléaire dans un article retentissant publié en 2001 [2]. Malgré l'opposition de quelques «irréductibles» [3], des études récentes sont venues conforter la réalité d'une telle synthèse protéique nucléaire.

\section{Un contrôle de qualité \\ élimine des ARNm défectueux}

L'apparition d'un codon «non-sens» ou stop prématuré (PTC, premature termination codon) dans la phase de lecture ouverte de la section codante d'un ARNm peut déclencher sa dégradation (un processus appelé NMD ou non-sense mediated decay) (Figure IB). Cependant les codons stop prématurés n'entraînent pas toujours une dégradation de I'ARNm, parfois les ARN pré-messagers défectueux s'agrègent près de leur site de synthèse (Figure 1C) et parfois un épissage alternatif (NAS ou non-sense associated alternative splicing) élimine le codon stop (Figure 1D). De plus, les ARNm ne possédant pas de codons stop sont euxaussi dégradés (Figure $1 \varepsilon$ ). Un contrôle de qualité des ARNm permet d'éviter la synthèse de protéines tronquées, susceptibles de s'agréger et/ou d'agir comme des mutants dominants négatifs des protéines «normales» [4]. De ce fait, un contrôle de qualité des ARN rend compte de l'aspect récessif, à l'état hétérozygote, de nombreuses mutations introduisant des codons stop prématu$(\rightarrow) \mathrm{m} / \mathrm{s}$ rés. Ainsi, dès 1979, J.C. 2001, n०8-9, Chang et Y.W. Kan ont p.914 décrit un cas de $\beta$-tha-
Cnrs UMR 8541,

Régulation de l'expression

génétique, École Normale

Supérieure,

46 , rue d'Ulm,

75230 Paris Cedex 05,

France.

Bensaude@wotan.ens.fr

lassémie due à un PTC. La plupart des patients hétérozygotes sont phénotypiquement sains et ne synthétisent pas de protéine tronquée grâce à la NMD. Cependant, G.W. Hall et S. Thein ont décrit, en 1994, un cas de mutation PTC dans le dernier exon de la $\beta$-globine produisant un ARNm échappant à la NMD. On peut penser que l'accumulation d'une protéine défectueuse qui en résulte, est à l'origine d'une $\beta$-thalassémie sévère chez les patients hétérozygotes.

En l'absence de situation pathologique, la maturation des lymphocytes $B$ et $T$ s'accompagne de réarrangements «au hasard» des gènes codant pour les immunoglobulines ou les récepteurs des cellules T. On s'attend à ce que ces processus aboutissent fréquemment ( $66 \%$ des cas) à la production d'ARNm porteurs de codons stop prématurés qui doivent donc être éliminés [5]. D’une manière générale, le contrôle de qualité des ARNm semble concerner un très grand nombre de transcrits puisqu'en analysant les séquences des ADNc contenues dans les banques de données humaines (EST), B.P. Lewis et al. ont identifié 1106 gènes produisant des messagers porteurs de codons non-sens en amont d'une «épissure», donc susceptibles de NMD [6].

\section{Un contrôle nucléaire de la qualité} des ARNm

Dès les premiers travaux, il a été suggéré que, dans les cellules de mammifères, la 
dégradation des ARNm défectueux se produisait, au moins partiellement, dans le noyau. En effet, les codons stop prématurés, ou l'absence de codon stop, provoquent une baisse de la quantité d'ARNm matures co-purifiant avec les noyaux alors que ni la transcription du gène, ni la stabilité de l'ARNm cytoplasmique ne sont affectées. Inversement, des ARN prémessagers à l'origine d'ARNm porteurs de codons stop prématurés s'accumulent dans le noyau, au voisinage du site de leur transcription [7]. De plus, si l'on admet que l'épissage des ARN pré-messagers s'effectue dans le noyau, l'épissage alternatif NAS doit être un processus nucléaire. Cependant, le contrôle de qualité des ARNm peut aussi s'effectuer dans le cytoplasme, comme dans le cas de la $\beta$-globine [8]. Suivant les ARNm, le contrôle de qualité s'effectuerait soit dans le cytoplasme, soit dans le noyau.

\section{Des ribosomes bloqués déclenchent la dégradation des ARNm}

Très tôt on a avancé l'hypothèse d'une synthèse protéique pour rendre compte des observations sur le contrôle de qualité des ARNm. Après l'excision des introns, un complexe multiprotéique ( $\varepsilon J C$ ou exonjunction complex), résidu du complexe d'épissage, demeure associé au point d'épissure entre les deux exons épissés; ce complexe est éliminé par le passage du premier ribosome traduisant I'ARNm [9] (Figure 1A). Mais s'il existe un codon stop prématuré, à plus de 55 nucléotides en amont du point d'épissure, le complexe $\varepsilon J C$ reste en place. Le complexe $\varepsilon J C$ et le ribosome, «gelés» sur le codon stop, coopèrent ensuite pour déclencher la dégradation de cet ARN par NMD (Figure IB). Un ARNm présentant un codon stop en amont d'un site d'épissure est reconnu comme anormal et éliminé. Cela explique pourquoi les codons stop «normaux» sont généralement trouvés dans le dernier exon. Un mécanisme différent, impliquant lui aussi une synthèse protéique, serait responsable de la dégradation d'ARNm ne possédant pas de codon stop (Figure lE). Dans ce cas, le ribosome bloqué à l'extré- mité de l'ARNm s'associe au complexe «exosome» qui dégrade I'ARN par son activité exonucléase $3^{\prime}->5^{\prime}$ [10]. L'épissage alternatif NAS du messager du récepteur des cellules $T$ ne concerne que des sites placés en aval d'un codon AUG d'initiation de la traduction et ne se pro- duit pas en l'absence de codon AUG d'initiation ou lorsque la synthèse protéique est inhibée [11]. Cependant, des mécanismes de reconnaissance des codons stop prématurés ne faisant pas appel à la traduction pourraient rendre compte de certains cas de NAS [12].

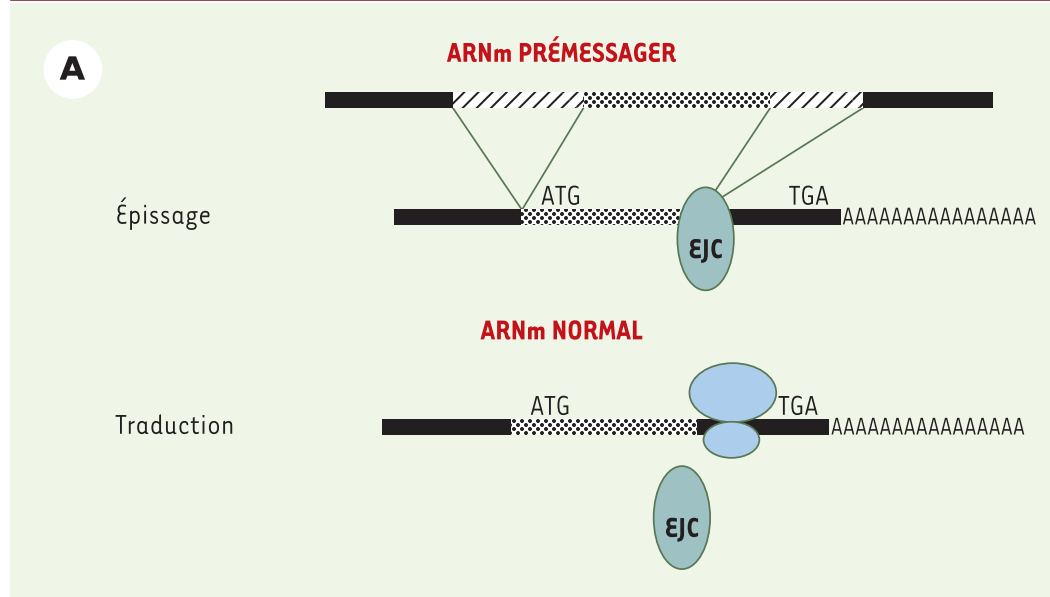

\section{ARNm PORTEUR D'UN CODON STOP PRÉMATURÉ}

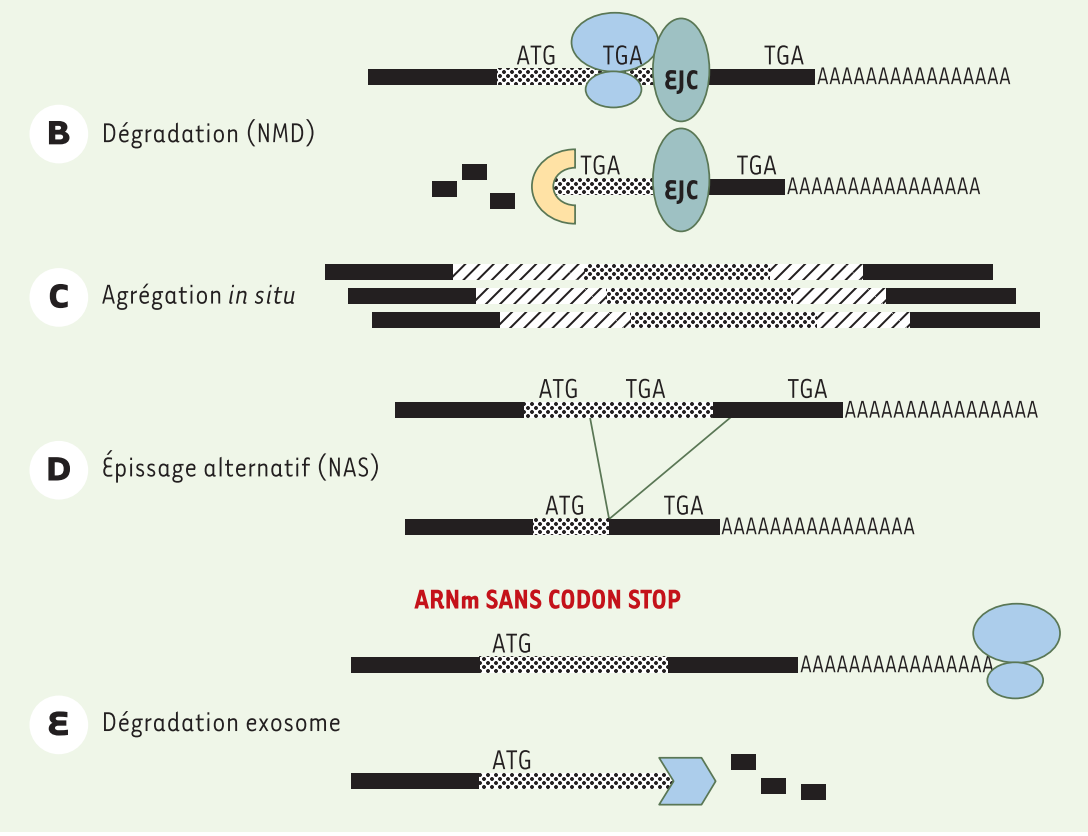

Figure 1. Mécanismes de contrôle de qualité des ARNm. Après l'épissage, un résidu du complexe d'épissage (le complexe $\varepsilon J C$ ) reste en place sur l'épissure. $A$. Si l'ARNm est «normal», le complexe $\varepsilon J C$ est chassé par le passage des ribosomes. Lorsqu'un codon stop «prématuré» précède l'épissure, les ribosomes restent en place et ne chassent pas le complexe $\varepsilon J C$. Plusieurs cas de figures sont observés. $B$. le complexe $\varepsilon J C$ demeuré en place recrute un système de dégradation des ARNm 5' -3' (non-sense mediated decay ou NMD). C. Les prémessagers «anormaux » s'agrègent près de leur site de transcription. $D$. Un épissage alternatif provoque la disparition du codon stop prématuré (non-sense mediated alternative splicing ou NAS). $\varepsilon$. En l'absence de codon stop, le ribosome progresse jusqu'au bout de la queue polyA où il demeure arrêté et recrute le complexe de l'exosome qui dégrade l'ARNm de 3'-5'. 


\section{Ribosomes}

\section{et facteurs de traduction}

sont recrutés au voisinage

des gènes actifs

Chez les eucaryotes, la synthèse protéique se décompose en plusieurs étapes: le recrutement d'un ribosome, la reconnaissance par celui-ci du premier codon (le plus souvent AUG, celui qui code pour la méthionine), l'allongement de la chaîne polypeptidique catalysé par le ribosome, l'arrêt de la synthèse lorsque le ribosome rencontre un codon stop. Les facteurs d'initiation de la traduction (elF) assistent les deux premières étapes, les facteurs d'élongation (eEF) interviennent lors de l'allongement des chaînes polypeptidiques, et les facteurs de terminaison (eRF) contribuent à l'arrêt et au détachement du ribosome de la protéine néo-synthétisée. Tous ces facteurs sont détectés dans le noyau, bien qu'en faibles quantités. L'observation la plus complète de ce genre vient d'être rapportée par S. Brogna du groupe de M. Rosbash qui étudiait les chromosomes polytènes des glandes salivaires de larves de drosophile [13]. Cette étude montre que les mêmes zones de transcription identifiables par un aspect de soufflé (puff) et par la présence d'ARN polymérase II sont marquées par des sondes ARN antisens de I'ARN ribosomique $18 \mathrm{~S}$ et par des anticorps dirigés contre 27 des sous-unités protéiques du ribosome ou contre le facteur d'initiation de la traduction, elF2, ou contre le facteur de terminaison de la traduction, eRF3. L'utilisation d'un gène inductible montre que le recrutement de ces protéines est détectable avant même que les transcrits n'aient pu être terminés. Tous les acteurs sont en place pour une traduction couplée à la transcription chez les eucaryotes comme chez les bactéries.

Les observations du groupe de J. Lis sur des chromosomes polytènes de drosophile vont plus loin et suggèrent un couplage entre la transcription et la dégradation des pré-messagers sans codon stop par l'exosome, puisque les composants de ce complexe sont recru- tés au niveau des gènes actifs par les facteurs généraux de transcription, Spt5 et Spt6 [14].

\section{Traduction nucléaire}

\section{d'ARNm naissants}

Les expériences de F. Iborra et P. Cook suggèrent fortement l'existence d'un couplage entre transcription et traduction dans des cellules humaines [2]. Ces auteurs ont très soigneusement isolé des noyaux et les ont incubés en présence de ARN de transfert chargés avec des acides aminés marqués; ceux-ci sont alors incorporés in situ dans des chaînes polypeptidiques. Or, cette incorporation in vitro d'acides aminés dans les noyaux est fortement stimulée par la présence des quatre ribonucléotides précurseurs des ARN. Inversement, elle est très fortement diminuée en présence d'inhibiteurs spécifiques de la transcription comme l' $\alpha$ amanitine. Ces observations suggèrent que les ARNm traduits sont ceux qui sont en cours de transcription. Le couplage de la traduction nucléaire à une transcription in situ rend les expériences de F. Iborra et $P$. Cook particulièrement convaincantes, car il écarte l'argument d'une contamination de leur préparation de noyaux par des polysomes cytoplasmiques. Plus récemment, A. Herbert et al. ont rapporté un cas spectaculaire de synthèse protéique nucléaire [15]. Ces auteurs ont empêché ou non la sortie de l'ARNm d'une protéine rapporteur. Puis, une utilisation très astucieuse de l'adénosine déaminase leur permet, en éliminant un codon stop, tantôt dans le noyau tantôt dans le cytoplasme, d'obtenir une synthèse du rapporteur dans un compartiment ou dans l'autre.

Pour conclure, la réalité d'une synthèse protéique nucléaire semble désor-

\section{RÉFÉRENCES}

1. Alberts B, Bray D, Lewis J, Raff M, Roberts K, Watson JD. Biologie moléculaire de la cellule, $2^{\mathrm{e}}$ éd. Paris: Flammarion MédecineSciences, 1992: 201.

2. Iborra FJ, Jackson DA, Cook PR. Coupled transcription and translation within nuclei of mammalian cells. Science 2001; 293: 1139-42.

3. Dahlberg JE, Lund $\varepsilon$ Goodwin EB. Nuclear translation: What is the evidence? RNA 2003; 9: 1-8.

4. Culbertson MR. RNA surveillance. Unforeseen consequences for gene expression, inherited genetic disorders and cancer. Trends Genet 1999. 15: 74-80.
5. Li S, Leonard D, Wilkinson MF. T cell receptor (TCR) mini-gene mRNA expression regulated by nonsense codons: a nuclearassociated translation-like mechanism. J Exp Med 1997 185: 985-92.

6. Lewis BP, Green RE, Brenner $S \varepsilon$. Evidence for the widespread coupling of alternative splicing and nonsense-mediated mRNA decay in humans. Proc Nat Acad Sci USA 2003 100: 189-92.

7. Muhlemann 0, MockCasagrande CS, Wang J, et al. Precursor RNAs harboring nonsense codons accumulate near the site of transcription. Mol Cell 2001; 8: 33-43.

8. Stevens A, Wang Y, Bremer K, et al. Beta-globin mRNA decay in erythroid cells: UG site-preferred endonucleolytic cleavage that is augmented by a premature termination codon. Proc Natl Acad Sci USA 2002; 99: 12741-6. 
9. Wagner $\varepsilon$, Lykke-Andersen J. mRNA surveillance: the perfect persist. J Cell $\mathrm{Sci}$ 2002; 115: 3033-8.

10. Maquat LE. Molecular biology. Skiing toward nonstop mRNA decay. Science 2002; 295: 2221-2.
11. Wang J, Chang YF, Hamilton J, Wilkinson MF. Nonsenseassociated altered splicing: a frame-dependent response distinct from nonsense-mediated decay. Mol Cell 2002; 10: 951-7.
12. Maquat $L \varepsilon$. Nasty effects on fibrillin pre-mRNA splicing: another case of ESE does it, but proposals for translation-dependent splice site choice live on. Genes Dev 2002; 16: 1743-53.

13. Brogna S, Sato TA, Rosbash M. Ribosome components are associated with sites of transcription. Mol Cell 2002; 10: 93-104.
14. Andrulis ED, Werner J, Nazarian A, ErdjumentBromage H, Tempst P, Lis JT. The RNA processing exosome is linked to elongating RNA polymerase II in drosophila. Nature 2002; 420: 837-41.

15. Herbert A, Wagner $S$, Nickerson JA. Induction of protein translation by ADARl within living cell nuclei is not dependent on RNA editing. Mol Cell 2002; 10: 1235-46.

\section{NOUVELLE}

\section{Réticulum endoplasmique, protéasome et maladies à prions}

Florence Béranger, Alain Mangé, Sylvain Lehmann

\author{
Institut de Génétique \\ Humaine, UPR CNRS1142, \\ 141, rue de la Cardonille, \\ 34396 Montpellier Cedex 5, \\ France. \\ sylvain.lehmann@igh.cnrs.fr
}

Chez l'homme, les encéphalopathies spongiformes subaiguës transmissibles (ESST), ou maladies à prions, forment un groupe d'affections neurodégénératives comprenant la maladie de Creutzfeldt Jakob (CJD), le kuru, le syndrome de Gerstmann-Sträussler-Scheinker (GSS) et I'insomnie familiale fatale (pour revue, voir [1]). Les prions sont constitués principalement, voire exclusivement, d'une protéine nommée $\operatorname{Pr} \mathrm{P}^{\mathrm{Sc}}$. Cette protéine correspond à la forme altérée et pathogène d'une protéine normale, la $\operatorname{PrPC}$, et est principalement détectée dans le cerveau de personnes atteintes d'ESST. Elle possède une conformation différente de la $\operatorname{Pr}^{C}$ et peut être mise en évidence grâce à ses propriétés biochimiques particulières: insolubilité dans les détergents et résistance aux protéases.

La $\operatorname{Pr} P^{C}$ est une glycoprotéine ubiquitaire exprimée plus particulièrement dans les neurones du système nerveux central. Au cours de sa biosynthèse, la $\operatorname{PrP}^{C}$ est modifiée de façon post-traductionnelle avant d'être ciblée vers la membrane plasmique, où elle est impliquée dans un cycle d'endocytose et de recyclage membranaire.

Les compartiments cellulaires où s'effectue la conversion de la $\operatorname{PrP}^{C}$ en $\operatorname{PrP}^{\mathrm{Sc}}$ sont très mal définis. Afin de comprendre le rôle des différentes étapes des mouvements intracellulaires de la $\mathrm{PrP}^{C}$ sur sa conversion en $\operatorname{Pr} \mathrm{P}^{\mathrm{Sc}}$, nous avons bloqué certaines de ces étapes en surexprimant certaines protéines Rab, membres de la superfamille des protooncogènes Ras, qui jouent chez les mammifères un rôle important au cours du transport vésiculaire dans les cellules eucaryotes [2]. Comme les protéines Ras, les protéines Rab existent dans la cellule dans deux états, une forme inactive liée au GDP et une forme biologiquement active liée au GTP. Afin d'interférer avec les mécanismes de transport intracellulaire de la $\operatorname{Pr} P^{C}$ dans des lignées de neuroblastome murin infec- tées par les prions, nous avons choisi d'utiliser d'une part la protéine Rab4, qui agit dans les processus de recyclage des récepteurs endocytés, et d'autre part la protéine Rab6, qui stimule le transport rétrograde, de l'appareil de Golgi vers le réticulum endoplasmique (RE) [3].

Nos résultats suggèrent que la conversion de la $\mathrm{PrP}^{\mathrm{C}}$ en $\mathrm{PrP}^{\mathrm{Sc}}$ s'effectue dans un compartiment intracellulaire, puisque le fait de bloquer le recyclage vers la membrane plasmique avec un mutant Rab4-GDP augmente la proportion de protéines converties. Par ailleurs, nous avons pu montrer que la stimulation du transport rétrograde vers le RE par des mutants Rab6 s'accompagne d'une accumulation de $\operatorname{Pr} \mathrm{P}^{\mathrm{Sc}}$, ce qui suggère fortement que la conversion a lieu au niveau de ce compartiment. Dans le $R E$, la $\mathrm{PrP}^{C}$ se trouverait en contact avec des PrP mal conformées qui provoqueraient sa conversion en protéine pathogène. Des molécules de 